\title{
Identification of a Major QTL and Candidate Gene Analysis of Salt Tolerance at the Bud Burst Stage in Rice (Oryza sativa L.) Using QTL-Seq and RNA-Seq
}

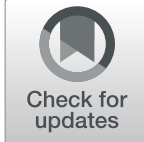

Lei Lei ${ }^{1 \dagger}$, Hongliang Zheng ${ }^{1,2 \dagger}$, Yanli Bi ${ }^{1}$, Luomiao Yang ${ }^{1}$, Hualong Liu' ${ }^{1}$, Jingguo Wang ${ }^{1}$, Jian Sun ${ }^{1}$, Hongwei Zhao ${ }^{1}$, Xianwei $\mathrm{Li}^{1}$, Jiaming $\mathrm{Li}^{1}$, Yongcai $\mathrm{Lai}^{2}$ and Detang Zou ${ }^{1 *}$ (i)

\begin{abstract}
Background: Salt stress is one of the main abiotic stresses that limits rice production worldwide. Rice salt tolerance at the bud burst stage directly affects the seedling survival rate and the final yield in the direct seeding cultivation model. However, the reports on quantitative trait locus (QTL) mapping and map-based cloning for salt tolerance at the bud burst stage are limited.

Results: Here, an $F_{2: 3}$ population derived from a cross between IR36 (salt-sensitive) and Weiguo (salt-tolerant) was used to identify salt-tolerant QTL interval at the bud burst stage using a whole-genome sequencing-based QTL-seq containing 40 extreme salt-tolerant and 40 extreme salt-sensitive individuals. A major QTL, GRSL7, related to relative shoot length (RSL) was detected on chromosome 7 using $\triangle S N P$ index algorithms and Euclidean Distance (ED) algorithms. According to single nucleotide polymorphisms (SNPs) between the parents, 25 Kompetitive allelespecific PCR (KASP) markers were developed near qRSL7, and regional QTL mapping was performed using 199 individuals from the $F_{2: 3}$ population. We then confirmed and narrowed down qRSL7 to a $222 \mathrm{~kb}$ genome interval. Additionally, RNA sequencing (RNA-seq) was performed for IR36 and Weiguo at $36 \mathrm{~h}$ after salt stress and control condition at the bud burst stage, and 5 differentially expressed genes (DEGs) were detected in the candidate region. The qRT-PCR results showed the same expression patterns as the RNA-seq data. Furthermore, sequence analysis revealed a $1 \mathrm{bp}$ Indel difference in Os07g0569700 (OsSAP16) between IR36 and Weiguo. OsSAP16 encodes a stress-associated protein whose expression is increased under drought stress.

Conclusion: These results indicate that OSSAP16 was the candidate gene of aRSL7. The results is useful for gene cloning of qRSL7 and for improving the salt tolerance of rice varieties by marker assisted selection (MAS).
\end{abstract}

Keywords: Oryza sativa L, Salt tolerance, QTL-seq, RNA-seq, Candidate gene

\footnotetext{
* Correspondence: zoudtneau@126.com

${ }^{\dagger}$ Lei Lei and Hongliang Zheng contributed equally to this work.

'Key Laboratory of Germplasm Enhancement, Physiology and Ecology of Food Crops in Cold Region, Ministry of Education, Northeast Agricultural University, Harbin 150030, China

Full list of author information is available at the end of the article
}

\section{Springer Open}

(c) The Author(s). 2020 Open Access This article is licensed under a Creative Commons Attribution 4.0 International License, which permits use, sharing, adaptation, distribution and reproduction in any medium or format, as long as you give appropriate credit to the original author(s) and the source, provide a link to the Creative Commons licence, and indicate if changes were made. The images or other third party material in this article are included in the article's Creative Commons licence, unless indicated otherwise in a credit line to the material. If material is not included in the article's Creative Commons licence and your intended use is not permitted by statutory regulation or exceeds the permitted use, you will need to obtain permission directly from the copyright holder. To view a copy of this licence, visit http://creativecommons.org/licenses/by/4.0/. 


\section{Background}

Salt stress is the main factor that restricts rice production because it reduces crop yield and limits agricultural land utilization (Hossain et al. 2015). Moreover, salinized soil has been increasing due to industrial pollution and unreasonable irrigation (Qadir et al. 2014). In recent years, the direct seeding method has become an important cultivation model due to the advantages of low labor intensity and high efficiency. Therefore, salt tolerance at the bud burst stage is a major factor that determines growth stability in salinized soil when adopting the direct seeding cultivation model in rice (Dingkuhn et al. 1992). Sensitivity of rice to salt stress at the bud burst stage leads to a decrease in seedling rate, resulting in lower yields (Zeng et al. 2001). Thus, studying the genetic mechanism of rice salt tolerance at the bud burst stage, and utilizing salt tolerance-related genes for breeding rice varieties suitable for direct seeding in salinized soil is of great importance.

Rice salt tolerance is a quantitative trait controlled by multiple genes, with a complex genetic mechanism (Zheng et al. 2015). To date, with the development of molecular marker technology and the construction of high-density linkage map, more than 900 rice salttolerant quantitative trait loci (QTLs) and 140 genes in response to salt stress have been identified on 12 chromosomes, mainly concentrated at the seedling stage and the field growth stage (http://gramene.org/). For instance, SKC1 (Ren et al. 2005), Saltol (Thomson et al. 2010), and DST (Huang et al. 2009) are the major genes associated with seedling stage under salt stress. There are few studies on salt-tolerance QTLs at the seed germination stage. In one case, an $F_{2: 9}$ recombinant inbred lines (RILs) population was used to analyze the imbibition rate and germination percentage of rice seeds under salt stress and control condition, and a total of 16 QTLs were detected, 4 of which explained more than $30 \%$ of the phenotypic variation (Wang et al. 2011). However, the reports on QTLs/gene mining for salt tolerance at the bud burst stage in rice are limited, and as rice seeds are often used after the germination stage by direct seeding in salinized soil, exploring the major QTL/gene of rice salt tolerance at the bud burst stage is of great significance to provide a theoretical and material foundation for their map-based cloning.

Compared to traditional QTL mapping, bulked segregant analysis (BSA) can effectively identify the molecular markers linked to genes or QTLs by generating two DNA bulks with significantly separated phenotypic traits (Michelmore et al. 1991). With the release of sequenced genomes, QTL-seq (Takagi et al. 2013) uses a combination of BSA and next-generation sequencing technologies to accelerate the identification of candidate genes without using markers or obtaining genotyping. To date, many loci have been identified by QTL-seq in different plants including arabidopsis (Schneeberger et al. 2009), soybean (Song et al. 2017), wheat (Trick et al. 2012), sorghum (Han et al. 2015), tomato (Liu et al. 2019) and cucumber (Lu et al. 2014). In rice, several QTLs associated with salt tolerance have also been identified by QTL-seq, such as mutant lines from which an extremely salt-tolerant pool was selected after the heading stage, leading to the identification of the salt-tolerant gene, OsRR22 (Takagi et al. 2015). Two bulks were created using extreme phenotypes of rice yield traits at the reproductive stage under salt stress using the RIL population developed from CSR11/MI48 and 5021 polymorphic loci and 34 QTL regions were revealed by $50 \mathrm{k}$ SNP chip, in which 7 new salt tolerance QTLs were identified (Tiwari et al. 2016). These reports suggest that it is feasible to identify salt tolerant QTL/genes at the bud burst stage using QTL-seq.

Transcriptome analysis (RNA-seq) has high resolution and sensitivity, and can characterize the responses of various plant species to environmental stresses (Wang et al. 2009). Furthermore, RNA-seq can be used to find new transcripts and rare transcripts, and the data shows high reproducibility with respect to both technical and biological replications (Hoen et al. 2013). With the development of high-throughput sequencing technology, RNA-seq has been widely used to investigate the molecular mechanism of salt tolerance in rice (Shankar et al. 2016; Zhou et al. 2016), cotton (Guo et al. 2015), Leymus chinensis (Sun et al. 2013), and other crops ( $\mathrm{Hu}$ et al. 2015; Liang and Schnable 2016). However, thousands of differentially expressed genes (DEGs) are identified by RNA-seq, suggesting that it is still difficult to identify the target genes. Recently, a method of combining QTL-seq and RNA-seq was developed, and it can identify chromosome fragments or genes that can be verified by each other. Lately, a major QTL on chromosome 6 for capsaicinoid content was identified using QTL-seq and high-density genetic maps, and candidate genes were revealed by combining QTL-analysis and RNA-seq (Park et al. 2019). The gene, $q P H 7$, was identified in a $300 \mathrm{~kb}$ interval by QTL-seq in maize, and Zm00001d020874 was chosen as the target gene by RNA-seq (Zhang et al. 2019). AcPMS1, a candidate gene associated with DNA mismatch repair for the restorerof-fertility in onion was identified using BSA-seq and RNA-seq (Kim et al. 2015).

In this study, QTL-seq was employed to identify the QTLs for salt tolerance at the bud burst stage of rice by an $\mathrm{F}_{2: 3}$ population derived from a cross between IR36 (salt-sensitive) and Weiguo (salt-tolerant). A major QTL, $q R S L 7$, controlling relative shoot length (RSL) at the bud burst stage under salt stress was detected on chromosome 7. Regional linkage mapping analysis narrowed the 
QTL region down to a $222 \mathrm{~kb}$ interval, which contained 5 candidate genes that showed significantly different expression levels between the two parents as revealed by RNA-seq. The qRT-PCR and sequence analysis showed OsSAP16 was the candidate gene controlling the RSL in rice. This result will be useful for improving the salt tolerance and developing direct seeding of rice in salinized soil.

\section{Results}

\section{Screening and Evaluation of the Index for Two Mixed Pools}

In the present study, IR36 (salt-sensitive) and Weiguo (salt-tolerant) were crossed to develop an $\mathrm{F}_{2: 3}$ population containing 983 lines for QTL-seq analysis. The shoot length (SL), root number (RN), root length (RL), and their relative values in 983 individuals were evaluated under salt stress and control condition at the bud burst stage. Only the relative shoot length (RSL) showed a normal distribution, whereas the relative root number (RRN) and relative root length (RRL) exhibited skewed distribution (Fig. 1a), which was not in accordance with the inheritance of quantitative traits. More than 200 individuals showed the value of both RRN and RRL at 0 (Fig. 1a), which was due to slow or stopped root growth in most lines after salt stress. Thus, using RSL as the screening trait for the two mixed pools, 40 lines with extreme salt tolerance and 40 lines with extreme salt sensitivity were selected to prepare the tolerant pool (T-pool) and sensitive pool (S-pool), respectively, which were then used for DNA sequencing. The RSL were significantly lower in the S-pool than in the T-pool, indicating that the overall phenotypic data of the S-pool was greatly affected by salt stress (Fig. 1b). Moreover, the key to plant survival under $\mathrm{NaCl}$ salt stress is to maintain a low $\mathrm{Na}^{+} / \mathrm{K}^{+}$ratio in cells (Wang et al. 2012). To verify the accuracy of the selected extreme lines for T-pool and S-pool, we determined the $\mathrm{Na}^{+} / \mathrm{K}^{+}$ratio of shoots (SNK), and found that the SNK was lower in the T-pool than in the S-pool (Additional file 1: Figure S1). In addition, the phenotypic statistics of RRN and RRL also proved the exactitude of the extreme lines selection for the two pools (Fig. 1b). The phenotype of the two parents after salt treatment is displayed in Fig. 2, while Fig. 3 shows the phenotype of the $5 \mathrm{~F}_{2: 3}$ plants randomly selected from the T-pool and S-pool after salt treatment.

\section{Sequencing of the Parents and Extreme Pools}

A total of $141 \mathrm{Gbp}$ clean reads were obtained from the two parents and the two $\mathrm{F}_{2}$ pools, and the $\mathrm{Q} 30$ all reached $85 \%$. The average sequencing depth was $27.60 \mathrm{X}$, and the average mapped ratio and the average genome coverage was $98.95 \%$ and $96.21 \%$, respectively (Additional file 2: Table S1). GATK software was used to analyze the SNPs in the results. According to the SNPs obtained from the comparison of the parents and the two $F_{2}$ pools with Nipponbare reference genome, a total of $4,825,004$ SNPs were detected between IR36 and Weiguo, including 6395 non-coding transcript variants (Additional file 2: Table S2). In addition, 1,085,342 SNPs were detected between the T-pool and S-pool, and these contained 1740 noncoding transcript SNPs (Additional file 2: Table S2). An association analysis was performed using the SNPs detected between the T-pool and S-pool.

\section{QTL-Seq Analysis Combining SNP-Index Algorithms and ED Algorithms}

We used two algorithms to detect the QTL associated with salt tolerance at the bud burst stage. As shown in Fig. 4 and Table 1, a total of three regions were detected
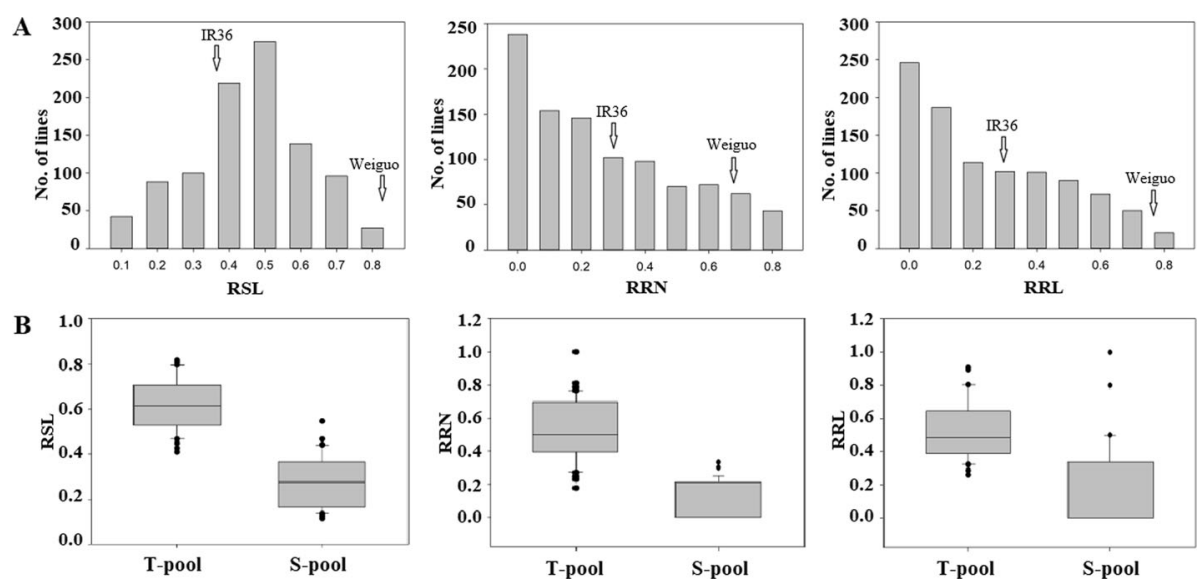

Fig. 1 a Phenotypic distribution of RSL, RRN and RRL of $983 \mathrm{~F}_{2: 3}$ lines. b Box-plot of phenotypic statistical of RSL, RRN and RRL in two pools. RSL: Relative shoot length; RRN: Relative root number; RRL: Relative root length. Arrows indicate the phenotypic value of IR36 and Weiguo 


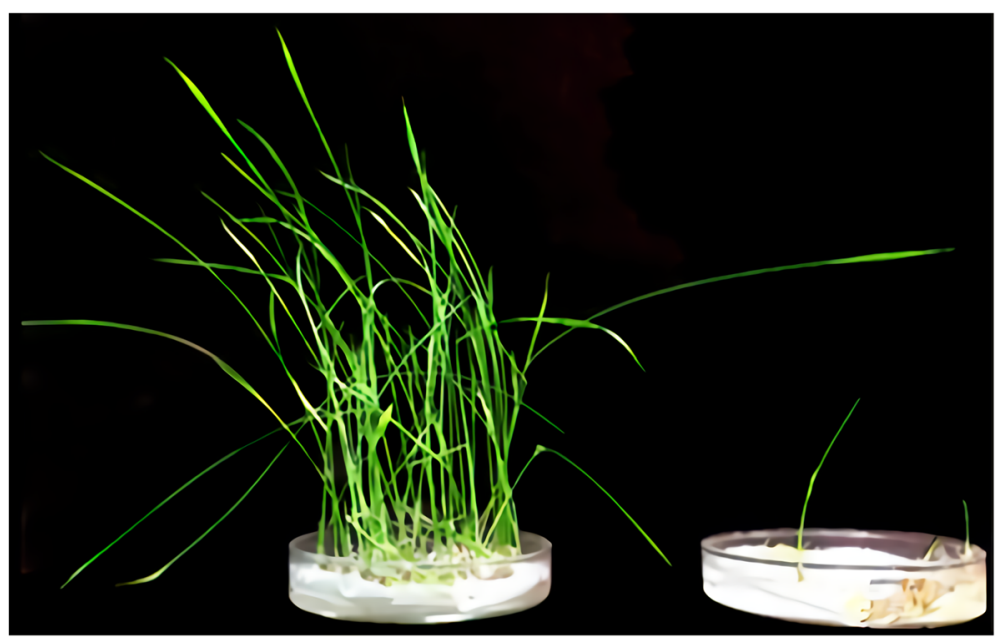

Weiguo

IR36

Fig. 2 The phenotype of Weiguo and IR36 after 10 days of $0.5 \% \mathrm{NaCl}$ treatment

by the $\triangle$ SNP-index method, which were located on chromosome 1,1 , and 7 , and the size of the regions was $2.68 \mathrm{Mb}, 2.92 \mathrm{Mb}$, and $4.17 \mathrm{Mb}$ respectively. A total of two regions were detected by the ED method, and both were located on chromosome 7 , and the physical distances were $5.17 \mathrm{Mb}$ and $14.13 \mathrm{Mb}$, respectively (Fig. 5, Table 1). By taking overlapping regions into account, these two methods yielded a region of $20,160,000-24$, 330,000 bp on chromosome 7, named $q R S L 7$, which was considered as the candidate region related to salt tolerance at the bud stage in rice (Table 1). Through analysis of the gene sequence in this interval, 16,415 SNPs and 2719 Indels were found, of which the number of SNPs and Indels causing amino acid changes was 554 and 50, respectively. Within the $4.17 \mathrm{Mb}$ region, 532 genes were predicted based on the RAP-DB database (http://rapdb. dna.affrc.go.jp/) (Additional file 2: Table S3).

\section{Regional Linkage Mapping Analysis}

As $q R S L 7$ still contained a large number of genes, we developed 25 KASP markers near this region and used them for genotyping $199 \mathrm{~F}_{2: 3}$ lines randomly selected

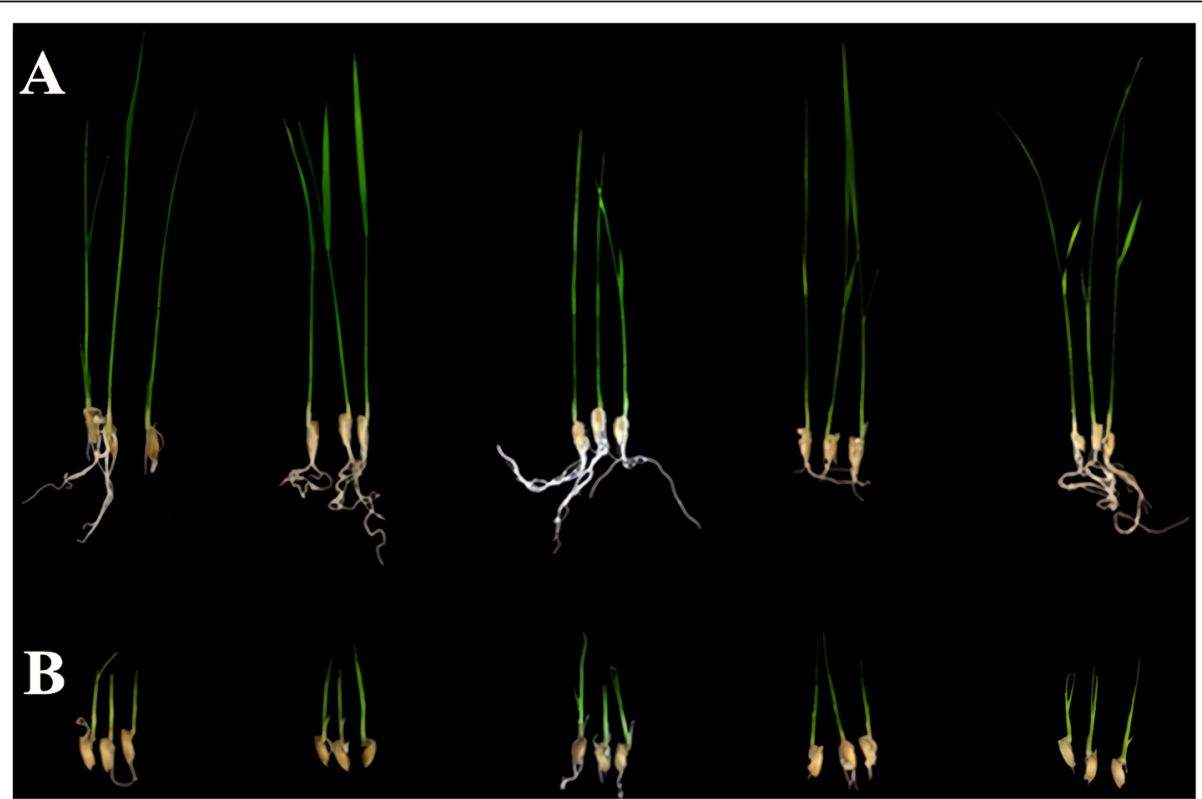

Fig. 3 a The phenotype of $5 \mathrm{~F}_{2: 3}$ plants randomly selected from T-pool after 10 days of $0.5 \% \mathrm{NaCl}$ treatment. $\mathbf{b}$ The phenotype of $5 \mathrm{~F}_{2: 3}$ plants randomly selected from S-pool after 10 days of $0.5 \% \mathrm{NaCl}$ treatment 

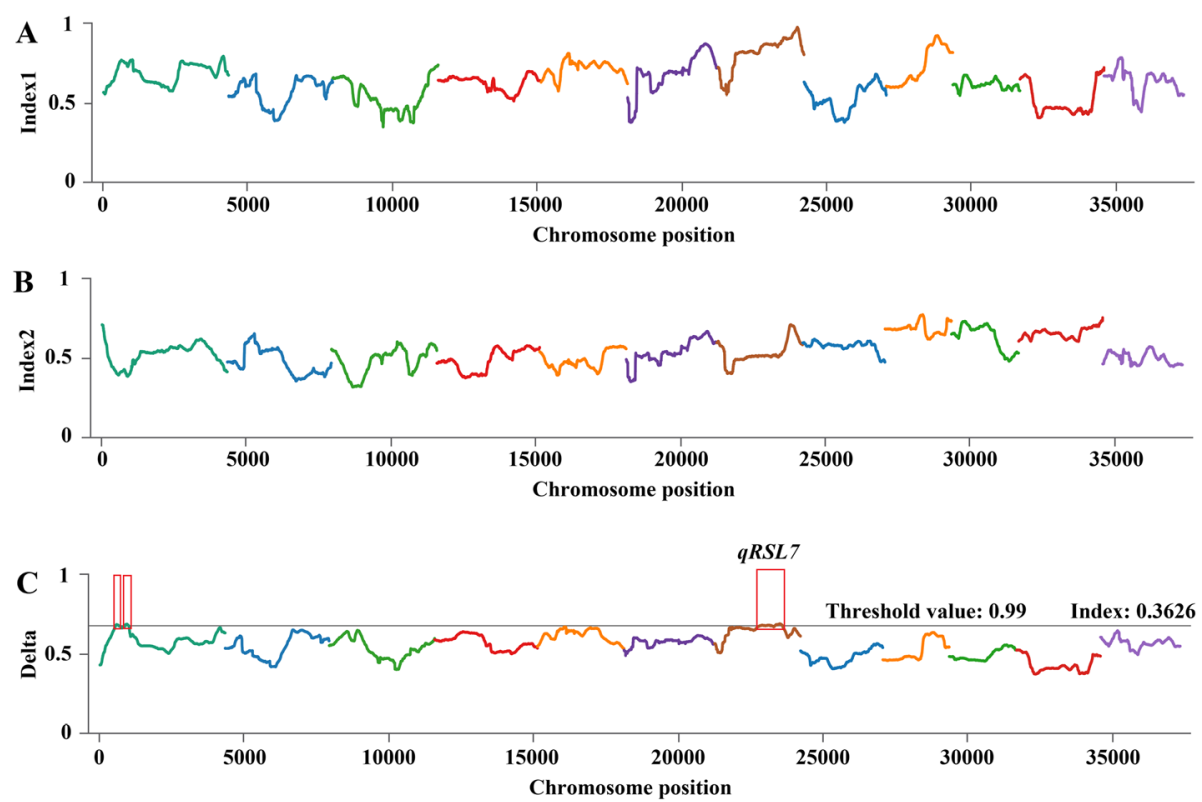

Fig. $4 \Delta S N P$-index algorithm mapping salt-tolerant QTL. X-axis represents the position of 12 chromosomes of rice and Y-axis represents the SNPindex or $\Delta S N P$-index. The different colour lines show the SNP-index or $\Delta$ SNP-index value of fitting results in 12 chromosomes. a The SNP-index graph of T-pool. b The SNP-index graph of S-pool. c The $\Delta S N P$-index graph

from 983 individuals of the $F_{2: 3}$ population. Under the condition of salt stress and control, the SL and its relative values of the parents and the $199 \mathrm{~F}_{2: 3}$ lines were counted (Additional file 2: Table S4). The phenotype values of SL under salt stress and control condition and RSL were all significantly different between the parents (Additional file 2: Table S4), and the $199 \quad \mathrm{~F}_{2: 3}$ lines showed continuous distribution under control and salt stress (Fig. 6). The parents' phenotype values were between the extreme values of the $199 \mathrm{~F}_{2: 3}$ individuals, which showed transgressive segregation (Fig. 6). The absolute values of skewness and kurtosis were both less than 1, indicating that the data of RSL were suitable for QTL analysis (Additional file 2: Table S4).

After calculation using ICIMapping4.2 software, a linkage interval of $222 \mathrm{~kb}$ was obtained on chromosome 7 (Fig. 7). As shown in Table 2, the RSL was linked to the SNP13-SNP14 marker interval. qRSL7 explained 24.90\% of the phenotypic variation, and the LOD value and additive effect was 11.84 and -0.12 , respectively. The

Table 1 Distribution of salt-tolerant region on chromosomes detected by $\Delta$ SNP-index and ED algorithms

\begin{tabular}{lllll}
\hline Method & Chromosome & Start $(\mathrm{bp})$ & End $(\mathrm{bp})$ & Size $(\mathrm{Mb})$ \\
\hline$\Delta$ SNP-Index & 1 & $4,590,000$ & $7,270,000$ & 2.68 \\
& 1 & $7,800,000$ & $10,720,000$ & 2.92 \\
& 7 & $20,160,000$ & $24,330,000$ & 4.17 \\
ED & 7 & $6,942,377$ & $12,109,211$ & 5.17 \\
& 7 & $12,547,138$ & $26,674,659$ & 14.13 \\
\hline
\end{tabular}

positive allele of the QTL was contributed by Weiguo (Table 2). Thus, the interval of $q R S L 7$ was optimized from $4.17 \mathrm{Mb}$ to $222 \mathrm{~kb}$ by constructing a genetic map and 27 genes were contained in this region (Additional file 2: Table S3).

\section{RNA-Seq Statistics}

To identify DEGs between the parents, we performed transcriptome analysis of IR36 and Weiguo at $36 \mathrm{~h}$ after salt stress and control condition at the bud burst stage. In total, 23.77 million, 23.95 million, 23.70 million, and 24.17 million clean data were obtained from the IR36 and Weiguo transcriptome libraries under the normal condition (control) (marked as IR and WG) and salt treatment (marked as TIR and TWG), respectively. The high-quality base $(\% \geq \mathrm{Q} 30)$ of each sample were all more than $88.88 \%$ (Additional file 2: Table S5). The alignment results showed that $75.82-82.38 \%$ of the clean reads from all twelve samples could be mapped to the reference genome. On average, approximately 34.45 (72.57\%) and 37.49 (77.92\%) million reads were uniquely mapped to the reference genome using TopHat for IR36 and Weiguo, respectively (Additional file 2: Table S6).

\section{Classification of DEGs}

Four comparative groups (TWG vs. TIR, WG vs. IR, IR vs. TIR, and WG vs. TWG) were constructed by comparing the same rice cultivar under different conditions (control and stress) and the samples of different rice cultivars (IR36 and Weiguo) under the same condition. 


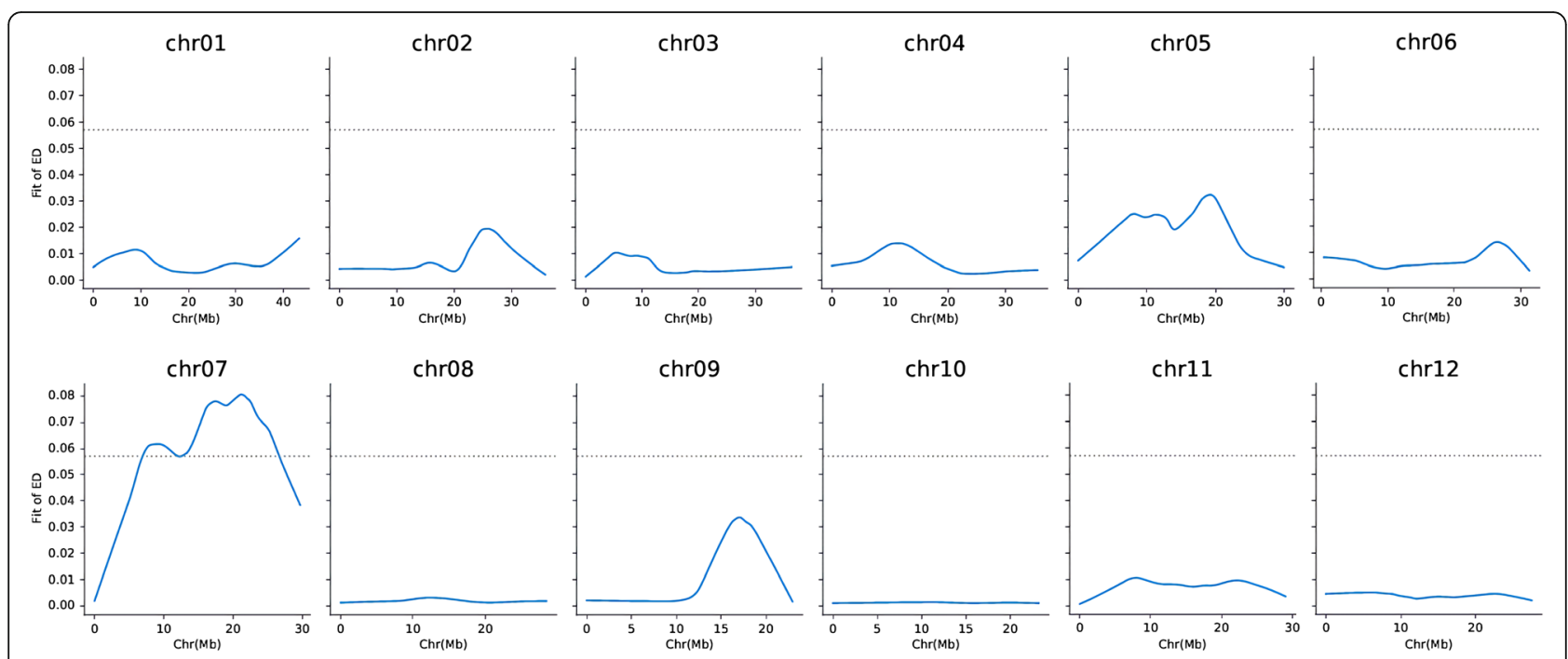

Fig. 5 ED algorithm mapping salt-tolerant QTL. The blue line represents the fitting value of ED, and the dashed line represents the threshold line

Additional file 3: Figure S2 shows the volcano plots of DEGs in the four groups. By restricting $-\log 10($ FDR $)>$ 1.3 (FDR $<0.05$ ), there were a total of 3227, 4177, 5921, and 1519 significant DEGs in TWG vs. TIR, WG vs. IR, IR vs. TIR, and WG vs. TWG, respectively. The results revealed that there were significant differences in the gene expression level within the varieties as well as the treatments. Within the two comparison groups of different varieties, 1150 DEGs were up-regulated and 2077 DEGs were down-regulated among the 3227 DEGs in TWG vs. TIR, and there were 1220 and 2957 DEGs that were up-regulated and down-regulated, respectively, among the 4177 DEGs in WG vs. IR. Furthermore, 3984 DEGs were up-regulated and 1937 were down-regulated in IR vs. TIR while 1237 were up-regulated and 282 were down-regulated in WG vs. TWG (Additional file 3: Figure S2). Obviously, more DEGs were up-regulated in IR vs. TIR. In addition, more DEGs in the salt-sensitive variety responded to salt stress compared to those in the salt-tolerant variety under salt conditions (Additional file 3: Figure S2). This result showed that the effect of salt stress on the salt-sensitive variety was greater than that on the salt-tolerant variety. In total, 9708 unique DEGs were detected in all four groups. These DEGs could be divided into 15 disjointed subgroups, among them 6.19\% (601/9708), 10.88\% (1056/9708), 34.06\% (3307/9708), and $2.79 \%(271 / 9708)$ were group-specific DEGs in TWG vs. TIR, WG vs. IR, IR vs. TIR, and WG vs. TWG, respectively. Additional file 4: Figure S3 shows the Venn diagram of the 9708 unique DEGs in these four groups.

\section{Gene Ontology (GO) and Kyoto Encyclopedia of Genes and Genomes (KEGG) Pathway Enrichment Analysis}

For all DEGs in the four comparison groups, a total of 2383 (73.85\%), 3119 (74.67\%), 4842 (81.78\%), and 1317 (86.7\%) DEGs were assigned GO terms in TWG vs. TIR, WG vs. IR, IR vs. TIR, and WG vs. TWG, respectively. Obviously, DEGs identified in IR vs. TIR were assigned significantly higher GO terms than those in WG vs. TWG. The cellular protein metabolic process was the most significant in the biological process category, indicating that the rice bud under salt treatment had wide metabolic activities. In the cellular component category and molecular function category, the cell part and
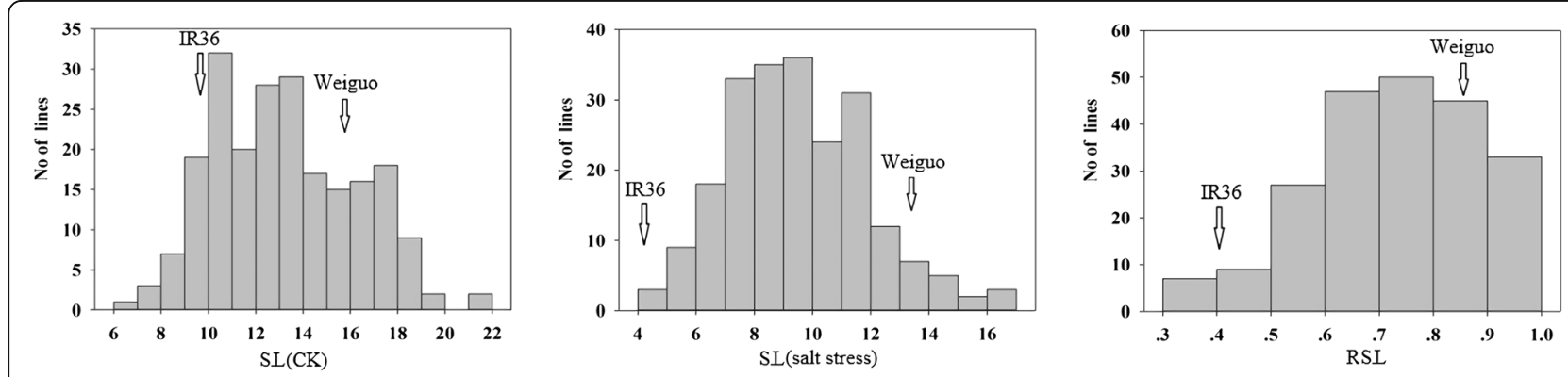

Fig. 6 Phenotypic distribution of SL under salt stress and CK and RSL of 199 F $2: 3_{3}$ lines. SL: Shoot length; RSL: Relative Shoot length; CK represents the lines growth in water at the bud burst stage. Arrows indicate the phenotypic value of IR36 and Weiguo 


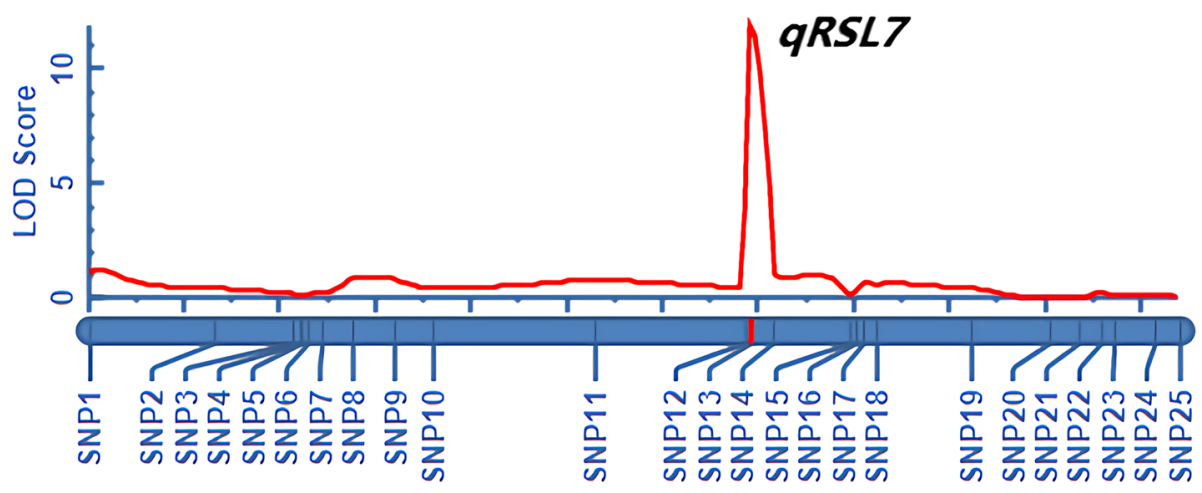

Fig. 7 qRSL7 detected in the $F_{2: 3}$ population contained 199 lines by QTL ICIMapping4.2 software

catalytic activity were the most significantly represented group, respectively (Additional file 5: Figure S4).

To further study the DEGs involved and enriched in various metabolic pathways, the KEGG pathway database was used for pathway-based analysis. The results revealed that 485 of 3227 DEGs in the TWG vs. TIR, 782 of 4177 DEGs in the WG vs. IR, 1407 of 5921 DEGs in the IR vs. TIR, and 401 of 1519 DEGs in the WG vs. TWG were annotated, respectively (Additional file 6: Figure S5). Among the significantly enriched pathways, the phenylpropanoid biosynthesis pathway contained more DEGs than the others. This result suggested that the phenylpropanoid biosynthesis pathway might have a modulating effect on salt-responsive gene expression. These annotations will provide valuable information for research on the salt stress response pathways in rice (Additional file 6: Figure S5).

\section{Candidate Gene Analysis}

QTL-seq analysis revealed a common QTL named qRSL7 (Table 1), and the interval was reduced from $4.17 \mathrm{Mb}$ to $222 \mathrm{~kb}$ by regional linkage mapping analysis (Fig. 7). Within the $222 \mathrm{~kb}$ region, 27 genes were predicted based on the RAP-DB database (http://rapdb. dna.affrc.go.jp/) (Additional file 2: Table S3). To detect the candidate genes, we combined the QTL-seq and RNA-seq results, and only selected the DEGs in IR vs. TIR and WG vs. TWG within the candidate region. Among the 27 predicted genes, 5 genes were differentially expressed under salt stress (Additional file 2: Table S7). Therefore, these 5 DEGs were selected as candidate genes for further confirmation. In addition, three genes, namely Os07g0569166, Os07g0569700, and Os07g0572075, were also differentially expressed in
WG vs. IR or TWG vs. TIR, and Os07g0570575 was significantly down-regulated in both WG vs. IR and TWG vs. TIR. In contrast, Os07g0570500 showed no significant up- or down-regulation in WG vs. IR or TWG vs. TIR (Additional file 2: Table S7).

To confirm the accuracy and reproducibility of Illumina RNA-Seq results, the 5 genes were compared for their expression levels between IR36 and Weiguo by quantitative real-time PCR (qRT-PCR) analysis under normal and salt stress conditions. The validation results for the 5 genes are shown in Fig. 8. Based on the RNA-seq results, the four genes Os07g0569166, Os07g0570500, Os07g0570575, and $0 s 07 g 0572075$ were all up-regulated in expression in TIR compared with IR. Moreover, Os07g0569700 was down-regulated in IR vs. TIR. There were no genes that were significantly differentially expressed in WG vs. TWG. The relative trends in the expression patterns of the qRT-PCR results were all consistent with the RNAseq data, but the absolute expression levels showed some differences (Fig. 8). Furthermore, to validate the expression of the 5 genes in the $F_{2: 3}$ population, we respectively selected 5 individuals with high, low and intermediate phenotype of RSL from the $199 \mathrm{~F}_{2: 3}$ population. Under the salt stress treatment, Os07g0569700 was down-regulated and the other 4 genes were up-regulated in low RSL lines, and no significant up- or down-regulation was observed in high and intermediate RSL lines (Fold change: expression data of salt treatment/expression data of control) (Additional file 7: Figure S6).

\section{Identification of Candidate Genes Responsible for Salt Tolerance of Rice}

To further obtain strong evidence to determine the most possible candidate gene, the 5 candidate genes were

Table 2 Detection information of aRSL7 detected by regional linkage mapping

\begin{tabular}{llllllll}
\hline Trait name & Chromosome & QTL & Left marker & Right marker & LOD & PVE(\%) & Add \\
\hline RSL & 7 & GRSL7 & SNP13 & SNP14 & 11.84 & 24.90 & -0.12 \\
\hline
\end{tabular}



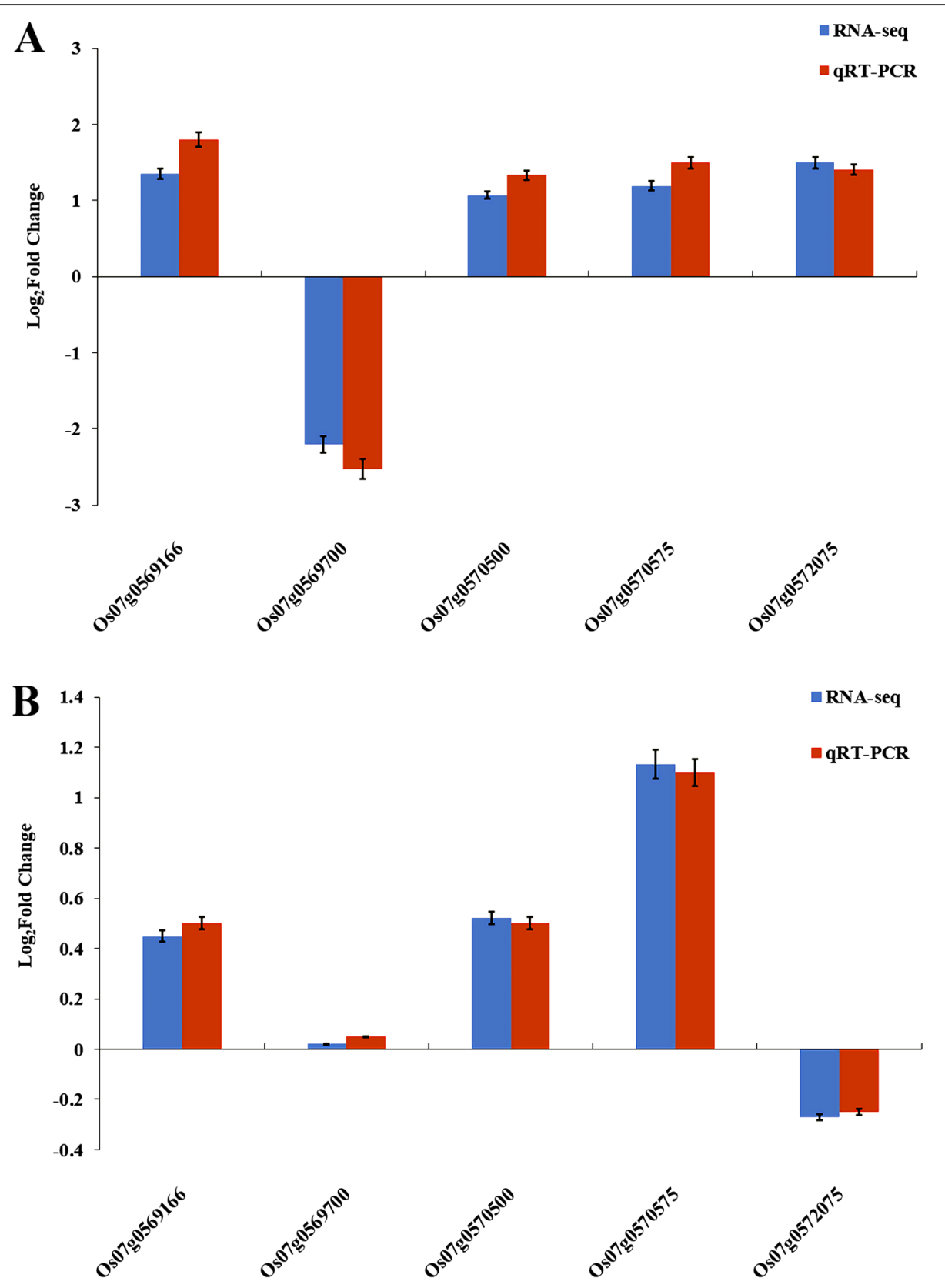

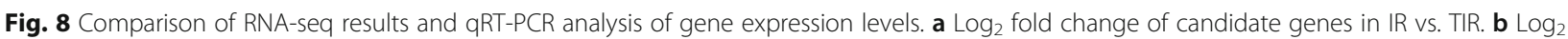
fold change of candidate genes in WG vs. TWG. Blue and red block represent RNA-seq results and qRT-PCR analysis, respectively

sequenced in IR36 and Weiguo. Sequence analysis exhibited no difference in the cDNA sequence and promoter region of Os07g0569166, Os07g0570575, and Os07g0572075 between IR36 and Weiguo. Os07g0570500 showed that in the CDS region, one SNP ( $\mathrm{T}$ to $\mathrm{C}$ ) was detected in IR36 compared with Weiguo (ATG start codon 96 bp downstream); however, we found that the SNP caused no amino acid changes using ProtParam (https://web.expasy.org/prot param/). Only Os07g0569700 showed that in the promoter region, IR36 contained a 1 bp insertion (ATG start codon
879 bp upstream) compared with Weiguo (Fig. 9). Next, we designed a KASP marker using the $1 \mathrm{bp}$ insertion sequence to genotype the two parents and 199 $\mathrm{F}_{2: 3}$ lines, which were also used for regional linkage mapping analysis. The KASP genotyping result is shown in Additional file 2: Table S8. The correlation analysis of female parent genotype, male parent genotype, and heterozygous genotype with RSL suggested that there was a significant positive correlation between the genotype and phenotype (Additional file 8: 


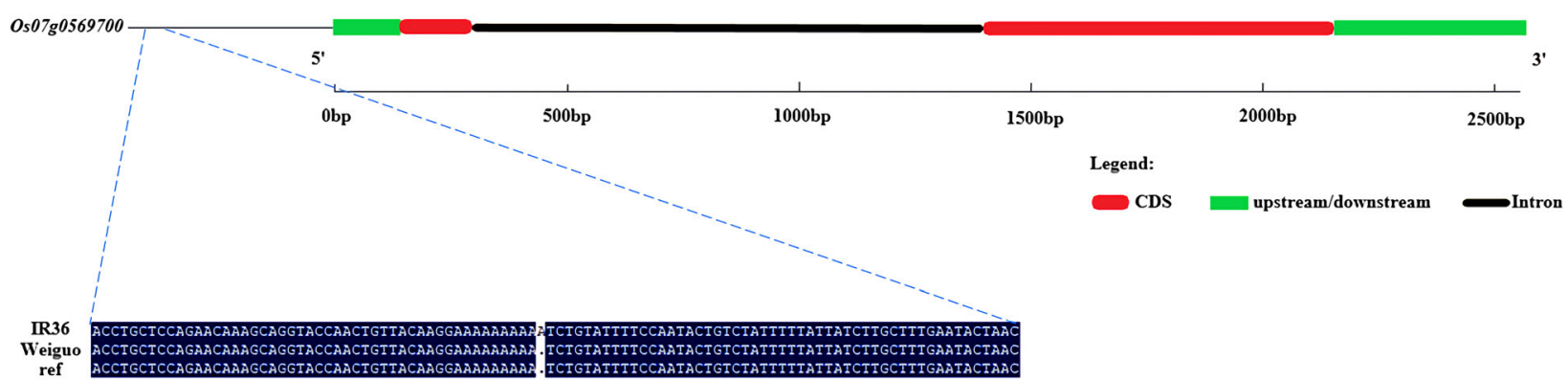

Fig. 9 Sequence difference analysis of Os07g0569700. The gene structure of Os07g0569700 and sequence differences in Os07g0569700 between IR36 and Weiguo. Ref is the reference sequence of Nipponbare genome

Figure S7). And, the results of ANOVA revealed that the differences between female parent genotype and male parent genotype, female parent genotype and heterozygous genotype, and male parent genotype and heterozygous genotype were all significant $(\mathrm{F}>\mathrm{F}$-crit, $P$ value $<0.01$; Additional file 2: Table S9). These suggested that the KASP maker was significantly associated with salt tolerance at the bud burst stage in the $\mathrm{F}_{2: 3}$ population under salt stress.

Therefore, Os07g0569700 was considered as the most possible functional gene underlying qRSL7. The candidate gene Os07g0569700 has been studied and named as OsSAP16 in a prior study (Wang et al. 2016). This gene encodes a stress-associated protein and its expression has been shown to increase under drought stress.

\section{Discussion}

Soil salt stress is one of the main abiotic stresses that affects crop yields, and seed germination, root length, and plant height is significantly inhibited under salt treatment (Liang et al. 2014). If rice plants are sensitive to salt stress at the bud burst stage, the seedling rate will be decreased, which will reduce the rice yield. However, the studies on QTLs/gene mining of rice after germination by direct seeding in salinized soil are limited. The salt tolerance of 295 rice germplasm resources was evaluated and extreme salt tolerance variety (Weiguo) /salt sensitive variety (IR36) were selected by our laboratory. Furthermore, IR36, an indica rice, is a recognized salt-sensitive variety that has been widely applied to the study of salt tolerance of rice (Khan et al. 2016; Yao et al. 2005). In the present study, an $\mathrm{F}_{2: 3}$ population derived from a cross between IR36 and Weiguo, a salt-tolerant japonica rice cultivar, was evaluated with respect to the SL, RN, and RL, and their relative values at the bud burst stage under salt stress and control (water) condition to collect individuals of two pools. The salt stress was induced using $0.5 \% \mathrm{NaCl}$ based on a preliminary experiment with 3 replicates, and the phenotypes of the lines treated with $0.5 \% \mathrm{NaCl}$ were found to be significantly different. All the measures used in this research could effectively obtain accurate SL, RN, and RL phenotypic data.

The traditional QTL fine mapping and map-based cloning are limited by high-density genetic map and a series of near-isogenic lines. For example, based on conventional mapping methods, 8 years were required for a predecessor to isolate the major salt-tolerant gene, qSE3 (He et al. 2019; Cheng et al. 2016). QTL-seq uses a combination of BSA and next-generation sequencing technologies to rapidly identify the chromosome region harboring the genes/QTLs of interest. For example, the salt-tolerant gene OsRR22 was isolated in an $\mathrm{F}_{2}$ population from a cross between the salt-tolerant mutant hst 1 and wild-type Hitomebore using BSA-Seq analysis, which only required 2 years (Takagi et al. 2015). In this study, we identified a major QTL (qRSL7) using QTLseq and rapidly optimized it by regional linkage mapping analysis from $4.17 \mathrm{Mb}$ to $222 \mathrm{~kb}$ to explain $24.90 \%$ of the phenotypic variation. Therefore, our research provides an effective strategy for the rapid identification of major salt-tolerant QTL at the bud burst stage. Compared with previous researches, some loci for salt tolerance have been reported near/harboring qRSL7 in recent years. $q P H 7.1 \mathrm{~s}$, a $4.34 \mathrm{Mb}$ interval linked with plant height at the reproductive stage under salt stress (Mohammadi et al. 2013), was overlapped with $q R S L 7$ in this study. qGP7-2 (Wang et al. 2011) and qSTS7 (Tian et al. 2011), two QTLs, identified to control the germination percentage and salt tolerance score, respectively, were approximately $1.0 \mathrm{~kb}$ and $29.3 \mathrm{~kb}$ distant to qRSL7; however, their phenotypic variation explanation (PVE) was only $9.2 \%$ and $10 \%$, respectively, which was much lower than that of $q R S L 7$ (24.90\%). These results indicate that of all the QTLs related to salt tolerance found on rice chromosome $7, q R S L 7$ is a major QTL related to salt tolerance at the bud burst stage in rice. Beyond these, we did not find any QTL loci close to our interval on chromosome 7.

Transcriptome analysis can be used to characterize various plant species responses to environmental stresses 
(Wang et al. 2009). In our study, 5 DEGs within the qRSL7 interval were found using RNA-seq (Additional file 2: Table S7). Among them, OsSAP16 showed a $1 \mathrm{bp}$ difference in the promoter region between IR36 and Weiguo and was significantly associated with the salt-tolerant phenotype. This gene encoded a $\mathrm{C} 2 \mathrm{H} 2$-type zinc finger protein and would be induced under drought stress (Wang et al. 2016). Furthermore, according to the transcriptome analysis and qRT-PCR results, the candidate gene OsSAP16 was significantly down-regulated after salt stress in IR vs. TIR (Fig. 8), revealing that the expression of this gene was inhibited by salt treatment in IR36.

To date, there have been no reports regarding OsSAP16 in rice salt tolerance. However, upon searching the Arabidopsis Information Resource (TAIR, http://www.arabidopsis.org/) database using the gene sequence of OsSAP16, two Arabidopsis homologous genes were identified (AT2G41835 and AT3G57480) (Additional file 2: Table S10), and only AT3G57480 was annotated with the function of "response to salt" in TAIR, suggesting that it may be involved in regulating the reactions to salt in Arabidopsis. Thus, we have a conclusion that OSSAP16 is the candidate gene for qRSL7.

In this study, IR36 and Weiguo are indica and japonica rice varieties, respectively. To prove that the $1 \mathrm{bp}$ Indel is specific for salt tolerance between indica and japonica rice varieties, we investigated the genotype of this $1 \mathrm{bp}$ Indel in 66 diverse rice accessions based on the data of pan-genome analysis (Zhao et al. 2018). The results showed that 16 of the 66 rice accessions contained this $1 \mathrm{bp}$ insertion, and among these, 15 rice accessions were O. sativa indica (including GP51, GP777-1, HP119, HP263, HP274, HP327, HP517-1, HP362-2, НP383, HP396, HP407, HP486, HP492, HP577, and GLA4) and the remaining rice accession was $O$. sativa aus, which was a subgroup of indica rice (Uraguchi et al. 2014); one remaining indica accession named W0128 was not detected any results (Additional file 2: Table S11). These results indicate that this $1 \mathrm{bp}$ insertion is probably specific to $O$. sativa indica and is associated with the decreased salt tolerance in IR36 at the bud burst stage. These data indicate that OSSAP16 is the strong candidate gene of $q R S L 7$. However, genetic transformation, and further studies are needed to confirm this conclusion.

\section{Conclusion}

In this study, QTL-seq, regional linkage mapping analysis, and transcriptome analysis were performed to identify the genes for salt tolerance at the bud burst stage in an $F_{2: 3}$ population derived from a cross between a salt-sensitive variety, IR36, and a salt-tolerant variety, Weiguo. A major QTL, qRSL7, associated with salt tolerance at the bud burst stage in rice was located at the $222 \mathrm{~kb}$ interval on chromosome 7 , which contained 5 candidate genes that showed significantly different expression between the two parents. Based on the qRT-PCR and sequence analysis, OsSAP16 was found to be the candidate gene controlling the RSL in rice. This study provides a fast and cost-effective strategy to identify salt-tolerant genes at the bud burst stage in rice.

\section{Methods \\ Plant Materials}

The two extreme rice cultivars, IR36 (salt-sensitive) as the female parent and Weiguo (salt-tolerant) as the male parent were crossed to harvest the $F_{1}$ population, and an $\mathrm{F}_{2}$ population was subsequently generated by selfing the $\mathrm{F}_{1}$ population. All plants were grown at the experimental station of Northeast Agricultural University. The leaves of $983 \mathrm{~F}_{2}$ lines collected at the active tillering stage, were stored at $-80^{\circ} \mathrm{C}$ for DNA extraction, and the corresponding $\mathrm{F}_{2: 3}$ seeds were collected for further experiments.

\section{Selection of Salt-Sensitive/Salt-Tolerant Individuals at the Bud Burst Stage}

Two DNA bulks for sequencing were constructed by selecting extreme individuals from the $\mathrm{F}_{2: 3}$ mapping population of 983 lines. Before this, 20 individuals were randomly selected from 983 lines to evaluate their salt tolerance together with the parents in $0.25 \%, 0.5 \%, 0.75 \%, 1 \% \mathrm{NaCl}$ and water (control), respectively. The phenotypes of the lines treated with $0.5 \% \mathrm{NaCl}$ were significantly different. Therefore, we selected $0.5 \% \mathrm{NaCl}$ to evaluate salt tolerance. The individuals were dried at $40{ }^{\circ} \mathrm{C}$ for 2 days to break the seed dormancy. Then, 120 full seeds which were randomly selected from each material, were divided into control and salt treatment on an average, and placed evenly in petri dishes. The seeds were then surface-sterilized with $2 \%$ $\mathrm{NaClO}_{3}$ for $10 \mathrm{~min}$ and rinsed thrice with sterile water. Next, the seeds were soaked in water at $30^{\circ} \mathrm{C}$ for germination, and when most seed buds were equal to half the length of the seed, 50 uniformly germinated seeds were selected in each petri dish and treated with $0.5 \% \mathrm{NaCl}$ or an equivalent amount of distilled water. The experiment was repeated three times. The temperature of the climatic cabinate was adjusted to $28{ }^{\circ} \mathrm{C} / 12 \mathrm{~h}$ (day) and $25^{\circ} \mathrm{C} / 12 \mathrm{~h}$ (night) with relative humidity set at $60 \%$. After 10 days of $0.5 \%$ $\mathrm{NaCl}$ treatment, 5 plants of each material were randomly selected to measure the SL, RN, and RL under the control and salt treatment condition. Then RSL, RRN, and RRL were further calculated to evaluate salt tolerance at the bud burst stage. Relative value $=$ (phenotype value under treatment condition) / (phenotype value under control condition). Moreover, the shoots of the selected extreme lines for T-pool and S-pool were harvested. The samples were dried at $80{ }^{\circ} \mathrm{C}$ for 2 days and then $0.1 \mathrm{~g}$ of each dried sample was ground and digested with $0.1 \mathrm{~N}$ Nitric acid (Fisher Scientific) at $70{ }^{\circ} \mathrm{C}$ for $8 \mathrm{~h}$ (Campbell et al. 2017). The 
concentrations of $\mathrm{Na}^{+}$and $\mathrm{K}^{+}$of shoots were analyzed by a flame photometer (Sherwood410, Cambridge, UK) and then SNK was further calculated.

\section{QTL-Seq Analysis}

Genomic DNA was extracted from the leaves of parent plants, IR36 and Weiguo, and from extreme individuals (40 extreme salt tolerance and 40 extreme salt sensitivity) in two pools using the cetyltrimeth-ylammonium bromide (CTAB) method with minor modifications (Pahlich and Gerlitz 1980). DNA quality was determined using the NanoPhotometer ${ }^{\circ}$ spectrophotometer (IMPL EN, CA, USA) with a required concentration greater than $50 \mathrm{ng} / \mu \mathrm{l}$. Next, the DNA was ultrasonically fragmented into $500 \mathrm{bp}$ using a Covaris S2 (Covaris) and was used for constructing DNA libraries with an NEBNext DNA Library Prep Reagent Set for Illumina (BioLabs). The DNA libraries were then sequenced on an Illumina HiSeq X Ten platform at the Beijing Genomics Institute (BGI). Clean reads from both parents and the two DNA pools were compared with the Nipponbare reference genome (Takuji 2005) using BWA software (Li and Durbin 2009). Reads of the T-pool and S-pool were separately aligned to Weiguo and IR36 consensus sequence reads to call SNPs with the SAM tools software (Li and Durbin 2009). PCR duplicates were removed using MarkDuplands tool in Picard, and GATK software was utilized for quality control and filtering to ensure the accuracy of SNPs. The SNP-index association algorithm was used for calculating the genotype frequency differences between two pools. A SNP index represented the proportion of reads containing the SNP different from the reference sequence. The $\triangle$ SNP-index of each locus was calculated by subtracting the SNPindex of the T-pool from that of the S-pool (Takagi et al. 2013). The ED algorithm was used to search markers with significant differences between the pools based on sequencing data and evaluation of relevant regions between the markers and traits. The equation of ED algorithm was as follows:

$$
E D=\sqrt{\left(A_{a a}-A_{a b}\right)^{2}+\left(C_{a a}-C_{a b}\right)^{2}+\left(G_{a a}-G_{a b}\right)^{2}+\left(T_{a a}-T_{a b}\right)^{2}}
$$

where $A_{a a}, C_{a a}, G_{a a}$ and $T_{a a}$ separately correspond to the frequency of bases $\mathrm{A}, \mathrm{C}, \mathrm{G}$ and $\mathrm{T}$ in the $\mathrm{T}$-pool. $\mathrm{A}_{\mathrm{ab}}$, $C_{a b}, G_{a b}$ and $T_{a b}$ separately correspond to the frequency of bases A, C, G and T in the S-pool. The depth of each base in two pools and the ED value of each SNP loci were calculated. To eliminate the background noise, the fit of ED was used as the associated value (Hill et al. 2013). The correlation interval was obtained by the $\triangle$ SNP-index algorithm and ED algorithm, and the common region associated with the two algorithms was the considered as the candidate region.

\section{Regional Linkage Mapping Analysis}

In total, 199 individuals were randomly selected from the $F_{2: 3}$ mapping population to evaluate salt tolerance by RSL, and the identification methods used were the same as those for the selection of salt-sensitive/salt-tolerant individuals. According to the SNPs near the QTL region obtained from QTL-seq, Primer 5.0 was used to design the 25 KASP markers (Additional file 2: Table S12) to genotype 201 individuals, including 199 from the $\mathrm{F}_{2}$ population and two parents. KASP assays were conducted in a 1536-well plate format using the protocol of LGC Genomics (LGC, Middlesex, UK) with the following PCR protocol: $94^{\circ} \mathrm{C}$ for $15 \mathrm{~min}, 95^{\circ} \mathrm{C}$ for $20 \mathrm{~s}, 55^{\circ} \mathrm{C}$ for $60 \mathrm{~s}\left(-1^{\circ} \mathrm{C} /\right.$ cycle, 10 cycles in total $)$ and $94^{\circ} \mathrm{C}$ for 10 s; $57^{\circ} \mathrm{C}$ for $60 \mathrm{~s}$ ( 30 cycles). The KASP reaction mixture system is shown in Additional file 2: Table S13 and the KASP reaction was set as described previously (Liu et al. 2019) with minor modifications. The Synergy H1 fullfunction microplate reader (FLUO star Omega, BMG Labtech, Germany) was used to read the fluorescence signal. Then, the linkage group was constructed by ICIMapping 4.2, and the grouping module LOD $>3.0$ was set to detect the linkage of the markers. Genetic distance was calculated using Kosambi function and the linkage map was drawn with MapChart2.2 (Voorrips 2002). QTL analysis was conducted by inclusive composite interval mapping (ICIM) in ICIMapping 4.2 (http:// www.isbreeding.net); $L O D \geq 3.0$ was selected as the threshold to determine QTL existence, and the significance level was set at 0.05 (Churchill and Doerge 1994).

\section{RNA Extraction and RNA-Seq}

The two parents, IR36 and Weiguo, were treated with $0.5 \% \mathrm{NaCl}$ at the bud burst stage, and the control was set at the same time. The buds of the treatment group and the control group ( 3 repeats) treated for $36 \mathrm{~h}$ were sampled and immediately frozen in liquid nitrogen. Total RNA was isolated from the buds using a TranZol Up RNA kit (TransGen Biotech). RNA purity was determined using the NanoPhotometer ${ }^{\circ}$ spectrophotometer (IMPLEN, CA, USA) and the A260/A280 ratios of all of samples were between 1.8 and 2.0. RNA integrity was detected using an Agilent 2100 Bioanalyzer, and no sign of degradation was found. cDNA was prepared from total RNA using the HiFiScript cDNA Synthesis Kit (CoWin Biosciences, Beijing, China). The cDNA library construction and Illumina sequencing were carried out following the manufacturer's recommendations (Illumina, San Diego, CA, USA) and the library quality was evaluated on an Agilent Bioanalyzer 2100 system. In total, 12 samples were sequenced. Clean reads were obtained by removing the adaptor sequences and low-quality sequences from the raw data, and mapping to the reference genome using TopHat v2.0.12 with the default parameters. The 
reference genome and gene model annotation files were downloaded from the Rice Genome Annotation Project (http://rice.plantbiology. msu.edu). All downstream analyses were based on clean data with high-quality reads. Differences in gene expression between the two samples were tested by the cuffdiff program. GO annotation of candidate genes was carried out using agriGO (Tian et al. 2017). Kyoto Encyclopedia of Genes and Genomes (KEGG) pathway enrichment analyses were performed using the KEGG database (http://www.genome.jp/kegg/).

\section{Quantitative Real-Time PCR Analysis and Candidate Gene Prediction}

Quantitative real-time PCR (qRT-PCR) was conducted to confirm the accuracy and reproducibility of the Illumina RNA-Seq results. qRT-PCR gene accessions and primer sequences are provided in Additional file 2: Table S14. Using $2 \times$ Fast qPCR Master Mixture (DINING, Beijing, China) on the Analytik Jena qTOWER system (German) to performed qRT-PCR. Three biological replicates, each with three technical replicates were examined per sample. Actin-EF $\alpha 1$ was used as the internal control. Relative gene expression levels were calculated using the $2^{-\Delta \Delta \mathrm{Ct}}$ method (Livak and Schmittgen 2000). The methods for RNA extraction and qRT-PCR of the 5 respective individuals with high, low, and intermediate phenotype of RSL from the $199 \mathrm{~F}_{2: 3}$ population were the same as above.

According to QTL-seq and linkage mapping analysis verification, a $222 \mathrm{~kb}$ QTL region on chromosome 7 related to salt tolerance at the bud burst stage was identified as the candidate region. Based on RNA sequencing data, the DEGs detected by transcriptome analysis in the candidate region were selected as the candidate genes.

\section{Candidate Gene Sequencing, Sequence Alignment and Validation of Candidate Genes with Molecular Markers} The corresponding candidate genes in Weiguo and IR36 were cloned using PCR and sequenced. With the genes in the Nipponbare genome as a reference, sequence alignment was carried out using DNAMAN software. The $1 \mathrm{bp}$ Indel difference between IR36 and Weiguo for the candidate gene Os07g0569700 was used to design the KASP marker (FAM primer: GAAG GTGACCAAGTTCATGCTGGTACCAACTGTTACA AGGAAAAAAAA; HEX primer: GAAGGTCGGA GTCAACGGATTGGTACCAACTGTTACAAGGAA AAAAAAA; Common primer: CCCGGTTAGTATTCAAAGCAAGAT). The marker was further used to genotype 201 individuals, including 199 lines from the $\mathrm{F}_{2: 3}$ population, which were also used for regional linkage mapping analysis, and the two parents. The method of the KASP assays was the same as that used in regional linkage mapping analysis.

\section{Supplementary information}

Supplementary information accompanies this paper at https://doi.org/10. 1186/s12284-020-00416-1.

Additional file 1: Figure S1. Box-plot of phenotypic statistical of SNK in two pools. SNK: $\mathrm{Na}^{+} / \mathrm{K}^{+}$ratio of shoots.

Additional file 2: Table S1. An overview of the QTL-seq results. Table S2. SNPs results between two parents and two mixed pools. Table S3. Annotated genes within the interval detected by QTL-seq. Table S4. Phenotypic variation of parents and F2:3 lines. Table S5. Summary of Illumina transcriptome reads mapped to the reference genome. Table S6. Statistics of transcriptome sequencing results. Table S7. RNA-seq data of the genes in candidate region. Table S8. The KASP genotyping results. Table S9. ANOVA results of three genotypes. Table S10. The Arabidopsis homologous genes of Os07g0569700. Table S11. The genotype of 1 bp Indel in 66 diverse rice accessions. Table S12. Primers of 25 KASP markers. Table S13. The KASP reaction system. Table S14. Primers for qRT-PCR in this study.

Additional file 3: Figure S2. Volcano plots for expressed genes in the four comparison groups. Volcano plots for all the expressed genes in (A) TWG vs. TIR, (B) WG vs. IR, (C) IR vs. TIR, and (D) WG vs. TWG. X- and Y-axis present the $\log 2$ (ratio) for the two samples and -log10 (FDR), respectively. Red (Up regulated) and green (down regulated) dots mean that the genes have significant difference, while the black dots correspond to genes with no significant differences.

Additional file 4: Figure S3. Venn diagrams for DEGs in the four comparison groups.

Additional file 5: Figure S4. The most significantly-enriched GO terms of DEGs from the four comparison groups. (A) TWG vs. TIR, (B) WG vs. IR, (C) IR vs. TIR, (D) WG vs. TWG.

Additional file 6: Figure S5. Analysis of KEGG enrichment for DEGs from the four comparison groups. (A) TWG vs.TIR, (B) WG vs. IR, (C) IR vs. TIR, (D) WG vs. TWG.

Additional file 7: Figure S6. The 5 genes expression assay in 5 individuals respectively selected from high, low and intermediate phenotype of RSL in199 $F_{2: 3}$ population. Fold change: expression data of salt treatment/expression data of control.

Additional file 8: Figure S7. The correlation analysis of female parent genotype, male parent genotype and heterozygous genotype with RSL.

\section{Abbreviations}

BSA: Bulked segregant analysis; QTL: Quantitative trait locus; RIL: Recombinant inbred line; ED: Euclidean distance; DEGs: Differentially expressed genes; InDel: Insertion deletion; KASP: Kompetitive allele-specific PCR; qRT-PCR: Real time quantitative polymerase chain reaction; SNPs: Single nucleotide polymorphisms; GO: Gene ontology; KEGG: Kyoto Encyclopedia of Genes and Genomes; PVE: Phenotypic variation explanation; S-pool: Sensitive pool; T-pool: Tolerant pool; SL: Shoot length; RN: Root number; RL: Root length; RSL: Relative shoot length; RRN: Relative root number; RRL: Relative root length; SNK: $\mathrm{Na}^{+} / \mathrm{K}^{+}$ratio of shoots

\section{Acknowledgments}

We would thank to the National Natural Science Foundation of China (31601377 and 31872884), the "Young Talents" Project of Northeast Agricultural University (17QC02) and the Heilongjiang Provincial government Postdoctoral Foundation of China (LBH-Z16188).

\section{Authors' Contributions}

$L L, H L Z$, and DT $Z$ conceived and designed the research. $Y L B, L M Y, H L L$ and JG W participated in data analysis. J S, HW Z, XW L, JM L and YC L performed material development, sample preparation and data analysis. $L L$ wrote the manuscript. DT $Z$ corrected the manuscript. The final manuscript was read and approved by all authors.

\section{Funding}

This study was supported by the National Natural Science Foundation of China (31601377), the "Young Talents" Project of Northeast Agricultural 
University (17QC02) and the Heilongjiang Provincial government Postdoctoral Foundation of China (LBH-Z16188).

\section{Availability of Data and Materials}

The BSA-seq clean data were uploaded in the NCBI Sequence Read Archive (NCBI SRA) under the accession number SRR10714357, SRR10714358, SRR10714420, SRR10724346. RNA-seq data were deposited to the SRA database of NCBI (SRP238139).

\section{Ethics Approval and Consent to Participate Not applicable.}

\section{Consent for Publication}

Not applicable.

\section{Competing Interests}

The authors declare that they have no competing interests.

\section{Author details}

${ }^{1}$ Key Laboratory of Germplasm Enhancement, Physiology and Ecology of Food Crops in Cold Region, Ministry of Education, Northeast Agricultural University, Harbin 150030, China. ${ }^{2}$ Heilongjiang Academy of Agricultural Sciences Postdoctoral Programme, Harbin 150030, China.

\section{Received: 13 January 2020 Accepted: 4 August 2020}

\section{Published online: 10 August 2020}

\section{References}

Campbell MT, Bandillo N, Al Shiblawi FRA, Sharma S, Liu K, Du Q, Schmitz AJ, Zhang C, Véry A-A, Lorenz AJ (2017) Allelic variants of OsHKT1; 1 underlie the divergence between indica and japonica subspecies of rice (Oryza sativa) for root sodium content. PLoS Genet 13:e1006823

Cheng J, He Y, Zhan C, Yang B, Xu E, Zhang H, Wang Z (2016) Identification and characterization of quantitative trait loci for shattering in japonica Rice landrace Jiucaiqing from Taihu Lake Valley, China. Plant Genome 9(3):1-9

Churchill GA, Doerge RW (1994) Empirical threshold values for quantitative trait mapping. Genetics 138:963-971

Dingkuhn M, De Datta SK, Javellana C, Pamplona R, Schnier HF (1992) Effect of late-season $\mathrm{N}$ fertilization on photosynthesis and yield of transplanted and direct-seeded tropical flooded rice. I. Growth dynamics. Field Crop Res 28: 223-234

Guo JY, Shi GY, Guo XY, Zhang LW, Xu WY, Wang YM, Su Z, Hua JP (2015) Transcriptome analysis reveals that distinct metabolic pathways operate in salt-tolerant and salt-sensitive upland cotton varieties subjected to salinity stress. Plant Sci 238:33-45

Han YC, Lv P, Hou SL, Li SY, Ji GS, Ma X, Du RH, Liu GQ (2015) Combining next generation sequencing with bulked segregant analysis to fine map a stem moisture locus in sorghum (Sorghum bicolor L. Moench). PLoS One 10(5): e0127065

He Y, Yang B, He Y, Zhan C, Cheng Y, Zhang J, Zhang H, Cheng J, Wang Z (2019) A quantitative trait locus, qSE 3, promotes seed germination and seedling establishment under salinity stress in rice. Plant J 97(6):1089-1104

Hill JT, Demarest BL, Bisgrove BW, Gorsi B, Su YC, Yost HJ (2013) MMAPPR: mutation mapping analysis pipeline for pooled RNA-seq. Genome Res 23 : 687-697

Hoen PACT, Friedlander MR, Almlof JC, Sammeth M, Pulyakhina I, Anvar SY, Laros JFJ, Buermans HPJ, Karlberg O, Brannvall M (2013) Reproducibility of highthroughput mRNA and small RNA sequencing across laboratories. Nat Biotechnol 31:1015-1022

Hossain H, Rahman MA, Alam MS, Singh RK (2015) Mapping of quantitative trait loci associated with reproductive-stage salt tolerance in Rice. J Agronomy Crop Sci 201:17-31

Hu LX, Li HY, Chen L, Lou YH, Amombo E, Fu JM (2015) RNA-seq for gene identification and transcript profiling in relation to root growth of bermudagrass ( Cynodon dactylon ) under salinity stress. BMC Genomics 16: 575-575

Huang XY, Chao DY, Gao JP, Zhu MZ, Shi M, Lin HX (2009) A previously unknown zinc finger protein, DST, regulates drought and salt tolerance in rice via stomatal aperture control. Genes Dev 23:1805-1817
Khan MSK, Saeed M, Iqbal J (2016) Quantitative trait locus mapping for salt tolerance at maturity stage in indica rice using replicated F2 population. Braz J Bot 39:641-650

Kim S, Kim C, Park M, Choi D (2015) Identification of candidate genes associated with fertility restoration of cytoplasmic male-sterility in onion (Allium cepa L.) using a combination of bulked segregant analysis and RNA-seq. Theor Appl Genet 128:2289-2299

Li H, Durbin R (2009) Fast and accurate short read alignment with burrowswheeler transform. Bioinformatics 25:1754-1760

Liang WJ, Cui WN, Ma XL, Wang G, Huang ZJ (2014) Function of wheat ta-UnP gene in enhancing salt tolerance in transgenic Arabidopsis and rice. Biochem Biophys Res Commun 450:794-801

Liang ZK, Schnable JC (2016) RNA-Seq based analysis of population structure within the maize inbred B73. PLoS One 11(6):e0157942

Liu G, Zhao TT, You XQ, Jiang JB, Li JF, Xu XY (2019) Molecular mapping of the Cf -10 gene by combining SNP/InDel-index and linkage analysis in tomato ( Solanum lycopersicum ). BMC Plant Biol 19:1-11

Livak K, Schmittgen T (2000) Analysis of relative gene expression data using realtime quantitative PCR and the 2- $\Delta \mathrm{C}$ t method. Methods 25:402-408

Lu HF, Lin T, Klein J, Wang SH, Qi JJ, Zhou Q, Sun JJ, Zhang ZH, Weng YQ, Huang SW (2014) QTL-seq identifies an early flowering QTL located near flowering locus T in cucumber. Theor Appl Genet 127:1491-1499

Michelmore RW, Paran I, Kesseli RV (1991) Identification of markers linked to disease-resistance genes by bulked segregant analysis: a rapid method to detect markers in specific genomic regions by using segregating populations. Proc Natl Acad Sci U S A 88:9828-9832

Mohammadi R, Mendioro MS, Diaz GQ, Gregorio GB, Singh R (2013) Mapping quantitative trait loci associated with yield and yield components under reproductive stage salinity stress in rice (Oryza sativa L.). J Genet 92:433-443

Pahlich E, Gerlitz C (1980) A rapid DNA isolation procedure for small quantities of fresh leaf tissue. Phytochemistry 19:11-13

Park M, Lee J, Han K, Jang S, Han J, Lim J, Jung J, Kang B (2019) A major QTL and candidate genes for capsaicinoid biosynthesis in the pericarp of Capsicum chinense revealed using QTL-seq and RNA-seq. Theor Appl Genet 132:515-529

Qadir M, Quillerou E, Nangia V, Murtaza G, Singh M, Thomas R, Drechsel P, Noble AD (2014) Economics of salt-induced land degradation and restoration. Nat Res Forum 38:282-295

Ren ZH, Gao JP, Li LG, Cai XL, Huang W, Chao DY, Zhu MZ, Wang ZY, Luan S, Lin HX (2005) A rice quantitative trait locus for salt tolerance encodes a sodium transporter. Nat Genet 37:1141-1146

Schneeberger K, Ossowski S, Lanz C, Juul T, Petersen AH, Nielsen KL, Jorgensen J, Weigel D, Andersen SU (2009) SHOREmap: simultaneous mapping and mutation identification by deep sequencing. Nat Methods 6:550-551

Shankar R, Bhattacharjee A, Jain MK (2016) Transcriptome analysis in different rice cultivars provides novel insights into desiccation and salinity stress responses. Sci Rep 6:23719-23719

Song J, Li Z, Liu ZX, Guo Y, Qiu LJ (2017) Next-generation sequencing from bulked-Segregant analysis accelerates the simultaneous identification of two qualitative genes in soybean. Front Plant Sci 8:919-919

Sun YP, Wang FW, Wang N, Dong YY, Liu Q, Zhao L, Chen H, Liu WC, Yin HL, Zhang XM (2013) Transcriptome Exploration in Leymus chinensis under Saline-Alkaline Treatment Using 454 Pyrosequencing. PLoS One 8(1):e53632

Takagi H, Abe A, Yoshida K, Kosugi S, Natsume S, Mitsuoka C, Uemura A, Utsushi H, Tamiru M, Takuno S (2013) QTL-seq: rapid mapping of quantitative trait loci in rice by whole genome resequencing of DNA from two bulked populations. Plant J 74(1):174-183

Takagi H, Tamiru M, Abe A, Yoshida K, Uemura A, Yaegashi H, Obara T, Oikawa K, Utsushi H, Kanzaki E (2015) MutMap accelerates breeding of a salt-tolerant rice cultivar. Nat Biotechnol 33(5):445-449

Takuji S (2005). The map-based sequence of the rice genome. Nature 436: 793-800.

Thomson MJ, Ocampo MD, Egdane J, Rahman MA, Sajise AG, Adorada DL, Tumimbang-Raiz E, Blumwald E, Seraj Zl, Singh RK (2010) Characterizing the Saltol quantitative trait locus for salinity tolerance in Rice. Rice 3:148-160

Tian L, Tan L, Liu F, Cai H, Sun C (2011) Identification of quantitative trait loci associated with salt tolerance at seedling stage from Oryza rufipogon. J Genet Genomics 38(12):593-601

Tian T, Liu Y, Yan HY, You Q, Yi X, Du Z, Xu WY, Su Z (2017) agriGO v2.0: a GO analysis toolkit for the agricultural community, 2017 update. Nucleic Acids Res 45:W122-W129

Tiwari S, Krishnamurthy SL, Kumar V, Singh B, Rao A, Sv AM, Rai V, Singh AK, Singh N (2016) Mapping QTLs for salt tolerance in Rice (Oryza sativa L.) by 
bulked Segregant analysis of recombinant inbred lines using 50K SNP Chip. PLoS One 11(4):e0153610

Trick M, Adamski NM, Mugford SG, Jiang CC, Febrer M, Uauy C (2012) Combining SNP discovery from next-generation sequencing data with bulked segregant analysis (BSA) to fine-map genes in polyploid wheat. BMC Plant Biol 12:14-14 Uraguchi S, Kamiya T, Clemens S, Fujiwara T (2014) Characterization of

OsLCT1, a cadmium transporter from indica rice (Oryza sativa). Physiol Plant 151:339-347

Voorrips RE (2002) MapChart: software for the graphical presentation of linkage maps and QTLs. J Hered 93:77-78

Wang F, Coe RA, Karki S, Wanchana S, Thakur V, Henry A, Lin HC, Huang JL, Peng SB, Quick WP (2016) Overexpression of OsSAP16 regulates photosynthesis and the expression of a broad range of stress response genes in Rice (Oryza sativa L.). PLoS One 11(6):e0157244

Wang Z, Gerstein M, Snyder M (2009) RNA-Seq: a revolutionary tool for transcriptomics. Nat Rev Genet 10:57-63

Wang Z, Wang J, Bao Y, Wu Y, Zhang H (2011) Quantitative trait loci controlling rice seed germination under salt stress. Euphytica 178(3):297-307

Wang ZF, Chen ZW, Cheng JP, Lai YY, Wang JF, Bao YM, Huang J, Zhang HS (2012) QTL analysis of $\mathrm{Na}+$ and K+ concentrations in roots and shoots under different levels of $\mathrm{NaCl}$ stress in rice (Oryza sativa L.). PLoS One 7(12):e51202

Yao MZ, Wang JF, Chen HY, Zhai HQ, Zhang HS (2005) Inheritance and QTL mapping of salt tolerance in Rice. Rice Sci 12:25-32

Zeng LH, Shannon MC, Lesch SM (2001) Timing of salinity stress affects rice growth and yield components. Agric Water Manag 48:191-206

Zhang HW, Wang X, Pan QC, Li P, Liu YJ, Lu XD, Zhong WS, Li MQ, Han LQ Li J (2019) QTG-Seq accelerates QTL fine mapping through QTL partitioning and whole-genome sequencing of bulked Segregant samples. Mol Plant 12(3):426-437

Zhao Q, Feng Q, Lu HY, Li Y, Wang A, Tian QL, Zhan QL, Lu Y, Zhang L, Huang T (2018) Pan-genome analysis highlights the extent of genomic variation in cultivated and wild rice. Nat Genet 50:278-284

Zheng HL, Zhao HW, Liu HL, Wang JG, Zou DT (2015) QTL analysis of Na+ and $\mathrm{K}+$ concentrations in shoots and roots under $\mathrm{NaCl}$ stress based on linkage and association analysis in japonica rice. Euphytica 201:109-121

Zhou Y, Yang P, Cui FL, Zhang F, Luo XD, Xie JK (2016) Transcriptome analysis of salt stress responsiveness in the seedlings of Dongxiang wild Rice (Oryza rufipogon Griff.). PLoS One 11(1):e0146242

\section{Publisher's Note}

Springer Nature remains neutral with regard to jurisdictional claims in published maps and institutional affiliations.

\section{Submit your manuscript to a SpringerOpen ${ }^{\circ}$ journal and benefit from:}

- Convenient online submission

- Rigorous peer review

- Open access: articles freely available online

High visibility within the field

- Retaining the copyright to your article

Submit your next manuscript at $\boldsymbol{\nabla}$ springeropen.com 